\title{
Three-Dimensional Structure of the Arp2/3 -Mediated Actin-Branch Junction
}

K. Anderson*, F.Beck**, M. Eibauer**, I. Rouiller, ${ }^{* 1}$ X.-P. Xu*, R. Li***, T. Pollard****, S. Nickell**, N. Volkmann*, and D. Hanein*

* Sanford-Burnham Medical Research Institute, 10901 N. Torrey Pines Rd., La Jolla, CA 92037

**Max-Planck-Institute of Biochemistry, Martinsried

***Stowers Institute for Medical Research, 1000 E. 50th Street, Kansas City, Missouri 64110 **** Yale University, New Haven, Connecticut 06520

The actin cytoskeleton provides both the force and the framework for cell motility, adhesion, and intracellular organization. We study macromolecular complexes involved in the assembly and regulation of the actin cytoskeleton at the leading edge of motile cells. Structural characterization of these multi-molecular protein complexes is likely to reveal potential mechanisms underlying cell growth, differentiation and migration, as well as the role of these interactions in tumor invasion and metastasis. Electron cryo-microscopy is the principal method for solving the structures of these large filamentous assemblies that remain beyond the reach of NMR and X-ray crystallography. Electron microscopy has grown to be a powerful imaging technique applicable to almost any kind of specimen, is parsimonious in its material requirements, and allows imaging of large macromolecular assemblies close to their physiological environment.

In my laboratory, we combine various electron cryo-microscopy, image analysis and bioinformatics approaches to extract high-resolution structural information of these large dynamic assemblies in their fully hydrated state. Here I will describe how a hybrid approach that involves a combination of electron tomography, image analysis, computational docking, biochemical and biophysical tools provided a detailed model of the Arp2/3-mediated actin branch junction at near-atomic resolution. Dynamic actin networks drive cell locomotion, phagocytosis, and intracellular motility of lipid vesicles, organelles, and invasive pathogens. Arp2/3 complex is the key component of these networks by virtue of its ability to initiate actin filament branches ("daughter filaments") on the sides of pre-existing ("mother") filaments. Our structural studies provide a model of tightly regulated branch formation in which Arp2/3 complex, nucleation promoting factors, a mother filament and actin monomers must act cooperatively to initiate branch formation, which in turn provide the force to allow the cell to move forward (1-4).

Although high-resolution structural approaches provide critical information about individual molecules and complexes, a barrier to progress remains their structural and functional 
integration at the cellular level. Towards this end we are currently developing techniques and protocols that allow us to image whole cells, in their fully hydrate state, and to use bioinformatics tools to correlate between the high-resolution structural information motives with the in situ characterization obtained from living cells. Here I will describe how electron cryo-tomography provides us the ability to localize these assemblies at the leading edge of migrating cells.

\section{References:}

1. Rouiller, I., et al., The structural basis of actin filament branching by the Arp $2 / 3$ complex. J Cell Biol, 2008. 180(5): p. 887-95.

2. Egile, C., et al., Mechanism of filament nucleation and branch stability revealed by the structure of the Arp2/3 complex at actin branch junctions. PLoS Biol, 2005. 3(11): p. e383. 3. Martin, A.C., et al., Effects of Arp2 and Arp3 nucleotide-binding pocket mutations on Arp2/3 complex function. J Cell Biol, 2005. 168(2): p. 315-28.

4. Volkmann, N., et al., Structure of Arp2/3 complex in its activated state and in actin filament branch junctions. Science, 2001. 293(5539): p. 2456-9.

${ }^{1}$ Current address, The Department of Anatomy and Cell Biology, Mc Gill University, 3640 University Street, Montreal, Quebec H3A 2B2 